\title{
Video Article \\ Hemogenic Endothelium Differentiation from Human Pluripotent Stem Cells in A Feeder- and Xeno-free Defined Condition
}

\author{
Ryo Ohta* ${ }^{1}$, Ryohichi Sugimura* ${ }^{1}$, Akira Niwa ${ }^{1}$, Megumu K. Saito ${ }^{1}$ \\ ${ }^{1}$ Center for iPS cell research and application (CiRA) \\ * These authors contributed equally
}

Correspondence to: Akira Niwa at akiranw@cira.kyoto-u.ac.jp, Megumu K. Saito at msaito@cira.kyoto-u.ac.jp

URL: https://www.jove.com/video/59823

DOI: doi:10.3791/59823

Keywords: Developmental Biology, Issue 148, hemogenic endothelium, hematopoiesis, human pluripotent stem cells, PSC spheroids, mesoderm organoids, directed differentiation, xeno-free, feeder-free

Date Published: 6/16/2019

Citation: Ohta, R., Sugimura, R., Niwa, A., Saito, M.K. Hemogenic Endothelium Differentiation from Human Pluripotent Stem Cells in A Feeder- and Xeno-free Defined Condition. J. Vis. Exp. (148), e59823, doi:10.3791/59823 (2019).

\section{Abstract}

Deriving functional hematopoietic stem and progenitor cells (HSPCs) from human pluripotent stem cells (PSCs) have been an elusive goal for autologous transplants to treat hematological and malignant disorders. Hemogenic endothelium (HE) is an amenable platform to produce functional HSPCs by transcription factors or to induce lymphocytes in a robust manner. However, current methods to derive HE are either costly or variable in yield. In this protocol, we established a cost-effective and precise method to derive HE within 4 days in a feeder- and xeno-free defined condition. The resultant HE underwent an endothelial-to-hematopoietic transition and produced $\mathrm{CD} 34^{+} \mathrm{CD} 45^{+}$clonogenic hematopoietic progenitors. The potential capacity of our platform for generating functional HSPCs will have broad applications in disease modeling and screening for therapeutic compounds.

\section{Video Link}

The video component of this article can be found at https://www.jove.com/video/59823/

\section{Introduction}

The development of new therapeutics for hematological and immunological disorders has been hampered by the lack of robust platforms for disease modeling and high throughput drug screening with autologous hematopoietic stem cells (HSCs) derived from patient pluripotent stem cells (PSCs). The breakthrough of induced (i)PSC technology holds promise to model diseases from patients' cells, but the establishment of functional HSCs from patient iPSCs has been elusive. In order to derive HSCs from human PSCs, the proper induction of embryonic patterning and transcriptional programs is key ${ }^{1,2,3,4,5,6,7,8}$. Recent progress in hematopoietic development has demonstrated the role of hemogenic endothelium (HE) in HSC development ${ }^{9}$. HE can be induced from PSCs and is amenable to downstream intervention such as gene transfer ${ }^{10,11,12,13}$. Using $\mathrm{HE}$ as a platform, the combination of 5 transcription factors (ERG, HOXA5, HOXA9, LCOR, RUNX1) successfully induced functional HSCs that engraft long-term and differentiate into multiple lineages ${ }^{14}$. However, variable and inefficient generation of HE from PSCs have hampered the systematic manipulation and analysis of cells along with the off-the-shelf production and large-scale induction of hematopoietic cells for clinical use.

Here, we describe a cost-effective and precise method to derive HE in 4 days with a feeder- and xeno-free defined condition. In this method, spheroid formation and spontaneous re-flattening on iMarix-511 ensures a consistent density of PSC colonies, which is crucial for the efficient induction of HE cells.

\section{Protocol}

\section{Reagents}

1. Cell lines (human PSCs): obtain either embryonic stem cells (ESCs) or iPSC lines 409B2 and 201B7(317-9)) and CBA11.

2. Reagent preparation

1. PSC plating medium: mix PSC maintenance medium with $10 \mu \mathrm{M} \mathrm{Y}-27632$ and store at $4{ }^{\circ} \mathrm{C}$

2. PSC Spheroid tiling medium: mix PSC maintenance medium with $625-1,250 \mathrm{ng} / \mathrm{mL}$ human recombinant Laminin-511 E8 fragment (LM511-E8)(depending on cell line) just prior to use.

NOTE: LM511-E8 can be mixed in media ${ }^{15}$. Other matrix such as full-length laminin-511 needs to be pre-coated, and even did so, they do not sustain mesodermal organoids adhered during differentiation process.

3. Day 0 differentiation medium: mix Mesodermal basal medium with $2 \mu \mathrm{M}$ CHIR99021, $80 \mathrm{ng} / \mathrm{mL}$ Bone morphogenetic protein 4 (BMP4) and $80 \mathrm{ng} / \mathrm{mL}$ Vascular endothelial growth factor (VEGF). 
4. Day 2 differentiation medium: mix Hemogenic basal medium with $1 \mu \mathrm{M}$ SB431542, $80 \mathrm{ng} / \mathrm{mL}$ VEGF and $100 \mathrm{ng} / \mathrm{mL}$ Stem cell factor (SCF).

5. Prepare phosphate buffer saline (PBS) without calcium or magnesium (See Table of Materials).

6. $1 \mathrm{mM}$ ethylenediaminetetraacetic acid (EDTA) buffer: add $1 \mathrm{~mL}$ of $0.5 \mathrm{M}$ EDTA to $500 \mathrm{~mL}$ of PBS.

7. EHT medium: mix Hematopoietic basal medium with $50 \mathrm{ng} / \mathrm{mL}$ SCF, $20 \mathrm{ng} / \mathrm{mL}$ thrombopoietin (TPO), $50 \mathrm{ng} / \mathrm{mL}$ Fms-related tyrosine kinase 3 ligand (FIt-3L), 20 ng/mL Interleukin-6/Interleukin-6 receptor alpha (IL-6/IL-6Ra), insulin-transferrin-selenium-ethanolamine, Lglutamine and Penicillin/Streptomycin/Amphotericin B.

8. FACS buffer: mix PBS with $2 \%$ fetal calf serum and $1 \mathrm{mM}$ EDTA.

9. Prepare magnetic separation buffer (See Table of Materials).

10. Prepare methylcellulose-based media (See Table of Materials).

\section{PSC Colony Formation}

1. Grow hPSCs to $70-80 \%$ confluency on a $0.5 \mu \mathrm{g} \mathrm{cm}{ }^{-2}$ LM511-E8-coated 6-well plate in $\mathrm{mTeSR} 1$ in a $37{ }^{\circ} \mathrm{C}$ incubator with $5 \% \mathrm{CO}_{2}$.

2. PSC dissociation (Day -4)

1. Aspirate the medium and wash the cells with PBS twice. Rinse the cells with dissociation solution. Incubate at $37^{\circ} \mathrm{C}$ for 15 min (cells will not desiccate in this time).

2. Suspend the cells in PSC maintenance medium, transfer the suspension to an appropriately sized tube and centrifuge the cells at 200 $\mathrm{x} g$ for $3 \mathrm{~min}$. Aspirate the supernatant and suspend the cells in PSC plating medium.

3. Plate the PSC suspension at a density of $48,000-70,000$ cells $\mathrm{cm}^{-2}$ (depending on the cell line) onto a micro-fabricated plastic vessel and incubate overnight in the $37^{\circ} \mathrm{C}$ incubator with $5 \% \mathrm{CO}_{2}$.

NOTE: We recommend $100 \mu \mathrm{L}_{\text {well }}{ }^{-1}$ of cell suspension in a 96-well micro-fabricated plastic vessel. You will typically seed 20,000 cells well ${ }^{-1}$ in a 96-well plate to yield approximately 100 spheroids.

4. Tiling PSC spheroids on LM511-E8 (Day -3)

1. Collect the PSC spheroids in a $15 \mathrm{~mL}$ conical tube by gently pipetting using P1000 micropipetter and leave the spheroids to stand at room temperature for 2 min to precipitate by gravity. Aspirate the supernatant.

2. Suspend in spheroid plating medium, dispense the suspension into a non-coated 6-well culture plate at a density of 4-spheroids $\mathrm{cm}^{-2}$ and culture in the $37^{\circ} \mathrm{C}$ incubator with $5 \% \mathrm{CO}_{2}$ for three days.

\section{Hemogenic Induction (Day 0)}

1. Aspirate the medium, add Day0 differentiation medium and culture in the $37^{\circ} \mathrm{C}$ incubator with $5 \% \mathrm{O}_{2}$ and $5 \% \mathrm{CO}_{2}$ (hypoxic incubator).

2. After two days of culture in Day0 differentiation medium, aspirate the medium, then add Day2 differentiation medium and culture in the hypoxic incubator.

\section{Isolation of Hemogenic Endothelium (Day 4)}

1. Two days later, aspirate the medium and wash the cells with PBS twice. Rinse the cells with dissociation solution. Incubate in the $37^{\circ} \mathrm{C}$ incubator with $5 \% \mathrm{CO}_{2}$ for $30 \mathrm{~min}$.

2. Gently suspend the cells in $1 \mathrm{mM}$ EDTA to dissociate to the single-cell level, transfer the suspension into a $50 \mathrm{~mL}$ conical tube and centrifuge the cells at $200 \times g$ for $3 \mathrm{~min}$. Aspirate the supernatant and suspend the cell pellet with $300 \mu \mathrm{L}$ of magnetic separation buffer.

3. Add $100 \mu \mathrm{L}$ of $\mathrm{CD} 34^{+}$microbeads and gently pipet. Incubate for $30 \mathrm{~min}$ at room temperature. Up to 20 million cells per $100 \mu \mathrm{L}$ CD $34^{+}$beads can be used.

4. Filter the suspension through a $40 \mu \mathrm{m}$ cell strainer

5. Separate the CD $34^{+}$cells using a magnetic separator.

\section{Induction of Endothelial-to-hematopoietic Transition (EHT)}

\section{Fibronectin coating}

1. Prepare the required volume of $5 \mu \mathrm{g} / \mathrm{mL}$ fibronectin-coating solution by diluting $1 \mathrm{mg} / \mathrm{mL}$ fibronectin in PBS.

2. Dispense $0.5 \mathrm{~mL}$ of the coating solution into each well of a 24 -well culture plate. Incubate the plate at room temperature for 30 min.

2. Centrifuge the sorted $\mathrm{CD} 34^{+}$cells at $200 \times \mathrm{g}$ for $3 \mathrm{~min}$. Resuspend the cell pellet in $1 \mathrm{~mL}$ of EHT medium

3. Determine the viable cell density with the cell counter. Adjust the cell density to 200,000 cells $\mathrm{mL}^{-1}$ by adding EHT medium.

4. Aspirate and discard the coating solution from the fibronectin-coated well. Dispense $0.5 \mathrm{~mL}$ of CD $34^{+}$cell suspension into each fibronectincoated well.

5. Incubate in the hypoxic incubator for one week.

\section{Flow Cytometry Analysis of Hematopoietic Cells}

1. Floating cell collection: Transfer the culture medium to a $15 \mathrm{~mL}$ conical tube. Centrifuge the medium at $200 \times g$ for 3 min. Aspirate the supernatant.

2. Adherent cell collection

1. Wash the cells with $0.5 \mathrm{~mL}$ of PBS twice. 
2. Rinse the cells with dissociating solution and aspirate the surplus liquid.

3. Incubate the plate in the $37^{\circ} \mathrm{C}$ incubator for $5 \mathrm{~min}$.

4. Resuspend the cells in $1 \mathrm{~mL}$ of FACS buffer.

3. Mix the floating cells and adherent cells.

4. Centrifuge the cells at $200 \times g$ for $3 \mathrm{~min}$. Aspirate the supernatant. Resuspend in $50 \mu \mathrm{L}$ of FACS buffer. Incubate the cells with anti-CD34 and anti-CD45 antibodies for $1 \mathrm{~h}$ at room temperature in the dark.

5. Wash the cells with PBS twice and centrifuge at $200 \times \mathrm{g}$ for $3 \mathrm{~min}$. Aspirate the supernatant and resuspend in $0.5 \mathrm{~mL}$ of FACS buffer with 0.5 $\mu \mathrm{g} / \mathrm{mL}$ 4',6-diamidino-2-phenylindole.

6. Measure CD34 and CD45 expression by flow cytometer.

\section{Colony Forming Unit (CFU) Assay of Hematopoietic Cells}

1. Hematopoietic cell collection: Transfer the medium from the culture to a $15 \mathrm{~mL}$ conical tube. Gently rinse the well twice with PBS.

2. Centrifuge the cells at $200 \times g$ for $3 \mathrm{~min}$. Aspirate the supernatant. Resuspend in $1 \mathrm{~mL}$ of EHT medium. Determine cell number with the cell counter.

3. Add a suspension volume equivalent of 10,000 cells to $3 \mathrm{~mL}$ of methylcellulose-based media supplemented with antibiotics and mix well 5 times with a $16 \mathrm{G}$ or $18 \mathrm{G}$ syringe.

4. Dispense the whole methylcellulose-based media suspension into each well of a 6-well plate.

5. Incubate in the $37^{\circ} \mathrm{C}$ incubator with $5 \% \mathrm{CO}_{2}$ for two weeks while keeping moist. Do not disturb the dish. Colonies are motion-sensitive.

6. After two weeks, count the colonies under a microscope.

\section{Representative Results}

A schematic of forming PSC colonies is depicted in Figure 1A. PSC spheroids are formed on a micro-fabricated plastic vessel for one day. As a representative result, 20,000 409B2 cells could be handled per 96-well of micro-fabricated plastic vessel, although other cell lines may require a higher density (30,000-40,000 cells per 96 -well of micro-fabricated plastic vessel). Those spheres are spontaneously flattened to a nearly twodimensional culture when plated onto a culture dish in the presence of LM511-E8.

A schematic of hemogenic induction is illustrated in Figure 1B. When PSC colonies grow to a diameter of $750 \mu \mathrm{m}$, the medium is sequentially changed to induce mesodermal organoids. The PSC colonies will gradually become a sunny side up structure during differentiation to mesodermal organoids by sequential medium change until Day 4. On Day 4, hemogenic endothelia are specified from mesodermal organoids by magnetic sorting and subsequently plated onto fibronectin in EHT medium. 5-10 million CD $34^{+}$CD $45^{-}$cells are expected from spheroids containing 1 million PSCs (Figure 1C).

It is observed that the cells morphologically change from endothelial to hematopoietic cells (Supplementary Video 1). A representative phasecontrast image of hematopoietic progenitor cells upon stimulation with hematopoietic cocktail on day 7 is shown in Figure 2A. Hematopoietic cell colonies emerged in the culture.

A representative flow cytometry plot is shown in Figure 2B. The whole culture is stimulated with hematopoietic cocktail and on day 7 is analyzed for the expression of CD34 and CD45 by flow cytometry. Hematopoietic progenitor cells express CD34 and CD45.

A CFU assay showed the generation of granulocytes/macrophage colonies from the CD $34^{+} \mathrm{CD} 45^{+}$hematopoietic progenitor cells (Figure $2 \mathrm{C}$ ). The estimated colony number is $38 \mathrm{CFU}-\mathrm{G} / \mathrm{M}$ per 10,000 cells (Figure 2D) 
A)

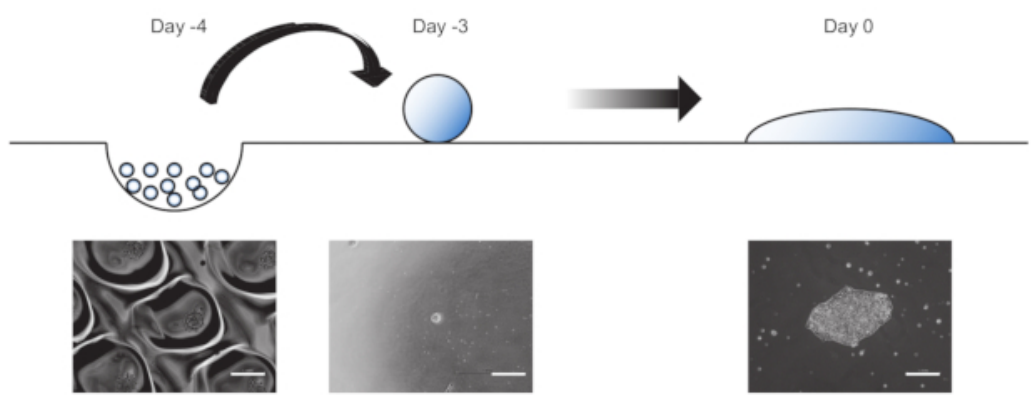

B)

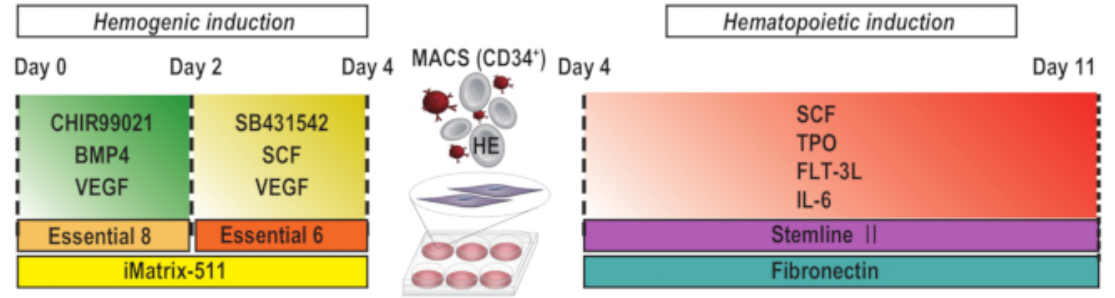

C)

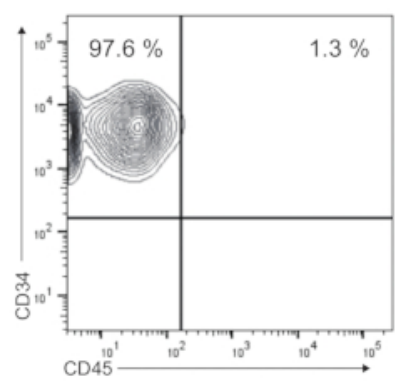

Figure 1: Schematic of tiling PSC colonies and subsequent hematopoietic differentiation via hemogenic endothelia. (A) Schematic process of forming PSC colonies. PSCs are maintained on a LM511-E8-coated 6-well plate in PSC maintenance medium. On Day -4, cells are detached and dissociated to the single-cell level using dissociating solution, subsequently plated onto micro-fabricated plastic vessel in PSC maintenance medium with Y-27632 and cultured overnight to form spheroids. On Day -3 , spheroids are plated in PSC maintenance medium with LM511-E8 and cultured for three days. By Day 0, spheroids are spontaneously flattened to a nearly two-dimensional culture. Scale bars $=200$ $\mu \mathrm{m}$. (B) On Day 0, the medium is replaced with Day0 differentiation medium and cultured in the hypoxic incubator. On Day 2 of the differentiation, the medium is replaced with Day2 differentiation medium. On Day 4 of the hemogenic endothelium enrichment, CD34 ${ }^{+}$cells are magnetically sorted and cultured on a fibronectin-coated 12-well plate in EHT medium for 6 days. (C) Representative flowcytometry plot of CD45 and CD34 expression of day 4 cells sorted by CD34. Please click here to view a larger version of this figure. 
A)

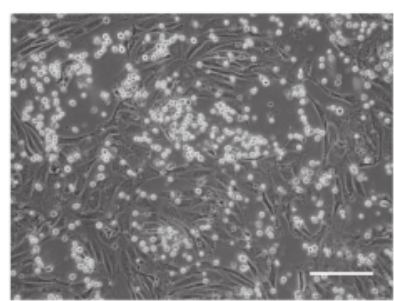

C)

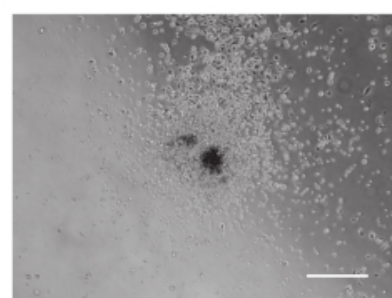

B)

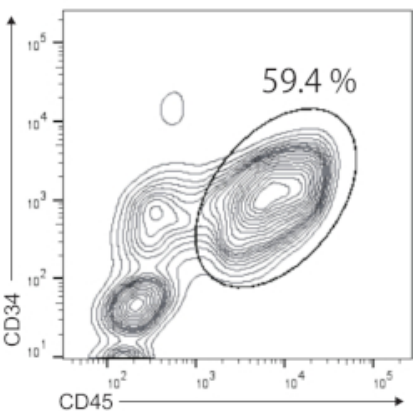

D)

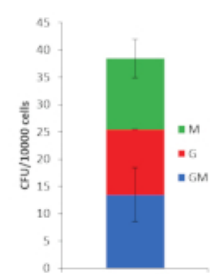

Figure 2: Analysis of hematopoietic property. (A) Representative phase-contrast image of hematopoietic progenitor cells after stimulation with hematopoietic cocktail on day 7 . Scale bar $=200 \mu \mathrm{m}$. (B) Representative flow cytometry plot of CD45 and CD34 expression of whole culture upon stimulation with hematopoietic cocktail on day 7. (C) Representative phase-contrast image of a granulocyte/macrophage colony generated from Day 11 hematopoietic progenitor cells. Scale bar $=500 \mu \mathrm{m}$. (D) Number of CFUs per 10,000 cells. Please click here to view a larger version of this figure.

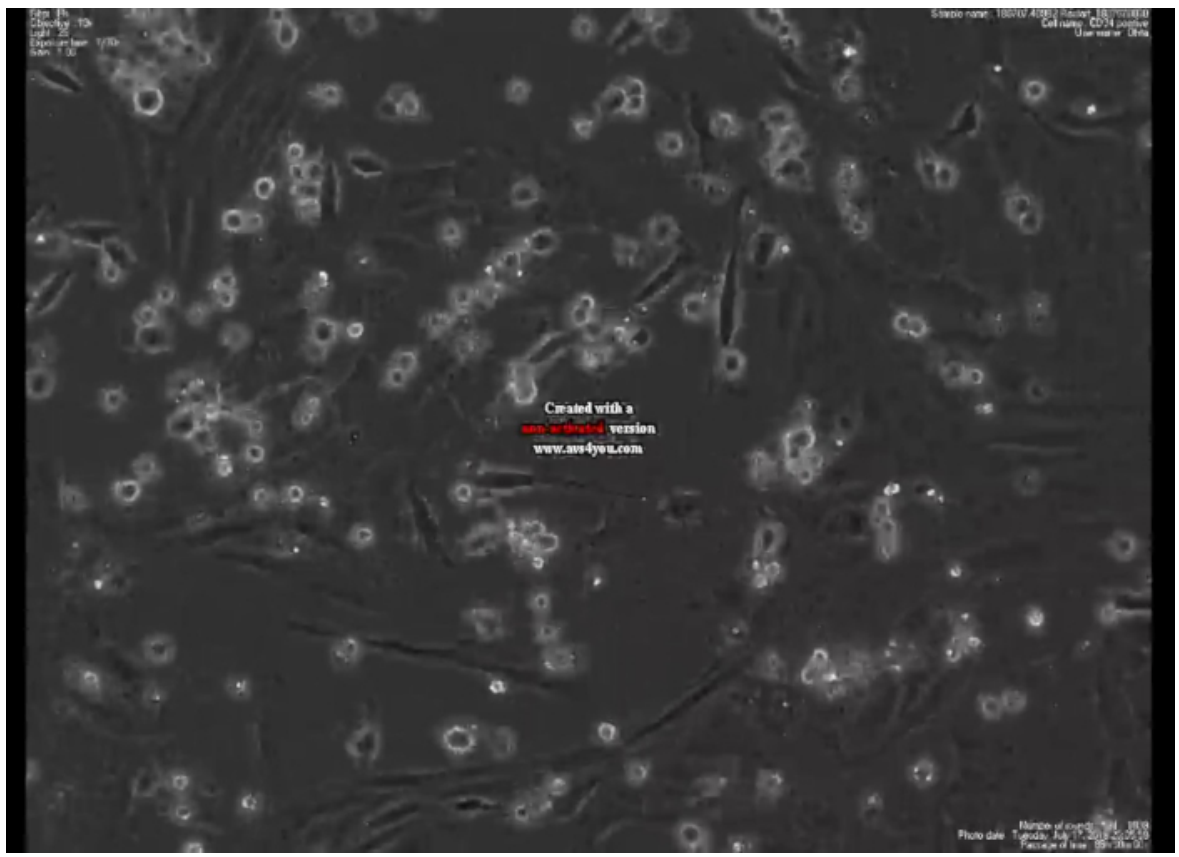

Supplementary video 1: EHT video. Please click here to download this file.

\section{Discussion}

Our feeder- and xeno-free defined induction method offers a precise and cost-effective platform to induce HE cells in a scalable manner. The faithful formation of hemogenic endothelium in conventional protocols ${ }^{10,11,12,13,14}$ have been hampered by lack of precise control of PSC colony density and size, which affects efficiency of morphogen/cytokine-based directed differentiation. We had experienced inconsistency of hemogenic endothelium induction in each independent batch from conventional protocols. The new protocol enables tight regulation of PSC colony density and size, thus overcomes the inconsistency of hemogenic endothelium induction. 
We exploited the uniformed formation of spheroids and subsequently conveyed a feeder- and xeno-free defined condition to form HE in a total of 4 days. We confirmed using three independent cell lines that the spheroid formation assures the consistent formation of HE cells. As a representative result, the 409B2 cell lines yielded $12.7 \%-23.6 \% \mathrm{CD} 34^{+}$cell efficiency based on three independent experiments. The critical factor to tile PSC spheroids is LM511-E8. We do not recommend substituting this with other matrix, such as Matrigel or full-length laminin products in our experiences. We experienced that mesodermal organoids were easily detached from Matrigel and lost during differentiation process. And neither Matrigel or full-length laminin do not anchor cells without pre-coating.

The potential limitation of this protocol is that we depend on PSC maintenance medium to maintain PSCs. We have tested other media such as StemFit, but we compromised hemogenic induction. Presumably cells were resistant to exit from pluripotent state in our short time (4 days) induction protocol. If one maintains PSCs in other media, we recommend adapting cells in PSC maintenance medium and conduct a couple of passages prior to differentiation. This platform will allow the systematic manipulation and analysis of cells, and the off-the-shelf production and large-scale induction of hematopoietic cells for potential clinical use. A long-term goal of this system is to model immunodeficiency diseases in humanized mouse models to investigate the responsible cellular and molecular mechanisms and conduct drug screenings.

\section{Disclosures}

The authors declare no conflicts of interest.

\section{Acknowledgments}

We are grateful to Alina Li and Seiko Benno for their technical assistance. We would also like to thank Ms. Harumi Watanabe for providing administrative assistance and Dr. Peter Karagiannis for editing the paper. This work was supported by the Core Center for iPS Cell Research of Research Center Network for Realization of Regenerative Medicine from the Japan Agency for Medical Research and Development (AMED) [M.K.S.], the Program for Intractable Diseases Research utilizing. Disease-specific iPS cells of AMED (17935423) [M.K.S.] and Center for Innovation program of Japan Science and Technology Agency (JST) [R.O. and M.K.S.].

\section{References}

1. Pereira, C. F. et al. Induction of a hemogenic program in mouse fibroblasts. Cell Stem Cell. 13, 205-218 (2013).

2. Lancrin, C. et al. The haemangioblast generates haematopoietic cells through a haemogenic endothelium stage. Nature. 457, $892-895$ (2009).

3. Loh, K. M. et al. Mapping the Pairwise Choices Leading from Pluripotency to Human Bone, Heart, and Other Mesoderm Cell Types. Cell. 166, 451-467 (2016).

4. Ng, E. S. et al. Differentiation of human embryonic stem cells to HOXA+ hemogenic vasculature that resembles the aorta-gonadmesonephros. Nature Biotechnology. 34, 1168-1179 (2016).

5. Batta, K. et al. Direct reprogramming of murine fibroblasts to hematopoietic progenitor cells. Cell Reports. 9, 1871-1884 (2014).

6. Sturgeon, C. M. et al. Defining the path to hematopoietic stem cells. Nature Biotechnology . 31, 416-418 (2013).

7. Ivanovs, A. et al. Human haematopoietic stem cell development: from the embryo to the dish. Development. 144, 2323-2337 (2017).

8. Blaser, B. W., Zon, L. I. Making HSCs in vitro: don't forget the hemogenic endothelium. Blood. (2018).

9. Speck, N. and Dzierzak, E. Of lineage and legacy: the development of mammalian hematopoietic stem cells. Nature imunology. (2008).

10. Niwa, A. et al. A novel serum-free monolayer culture for orderly hematopoietic differentiation of human pluripotent cells via mesodermal progenitors. PLoS One. 6, e22261 (2011).

11. Sturgeon, C. M. et al. Wnt signaling controls the specification of definitive and primitive hematopoiesis from human pluripotent stem cells. Nature Biotechnology. 32, 554-561 (2014).

12. Ditadi, A. et al. Human definitive haemogenic endothelium and arterial vascular endothelium represent distinct lineages. Nature Cell Biology. 17, 580-591 (2015).

13. Kennedy, M. et al. T lymphocyte potential marks the emergence of definitive hematopoietic progenitors in human pluripotent stem cell differentiation cultures. Cell Reports. 2, 1722-1735 (2012).

14. Sugimura, R. et al. Haematopoietic stem and progenitor cells from human pluripotent stem cells. Nature. 545, 432-438 (2017).

15. Miyazaki, T. et al. Efficient Adhesion Culture of Human Pluripotent Stem Cells Using Laminin Fragments in an Uncoated Manner. Scientific Reports . 7, 41165 (2017). 